\title{
Tratamiento resectivo con cierre primario mediante colgajo de Dufourmentel en la enfermedad pilonidal sacrococcígea (EPS)
}

\author{
Melina Colman ${ }^{1}$, Rocío Perez Dominguez ${ }^{2}$, Sergio Schlain ${ }^{3}$ \\ ${ }^{1}$ Residente de Cirugía General. HIGA San Roque de Gonnet, La Plata, Buenos Aires, Argentina. \\ ${ }^{2}$ Staff de la División Coloproctología HIGA San Roque de Gonnet, La Plata, Buenos Aires, Argentina. \\ ${ }^{3}$ Coordinador de la División Coloproctología del HIGA San Roque de Gonnet, La Plata, Buenos Aires, Argentina. \\ División de Coloproctología del HIGA San Roque de Gonnet, La Plata, Buenos Aires, Argentina.
}

\begin{abstract}
RESUMEN
Introducción: La enfermedad pilonidal sacrococcígea (EPS) una afección frecuente y que aparece descripta por primera vez por mayo en 1833. Su curso es benigno, el 0,1\% se malignizan a carcinoma escamoso. Presenta en su seguimiento variabilidad en el número de recidivas, según las series y el tipo de cirugía y una morbilidad alta también asociada al procedimiento. Se describen un sinnúmero de técnicas para su resolución.

Objetivo: Mostrar la utilización del cierre mediante colgajo de Dufourmentel como alternativa válida para el tratamiento de la enfermedad pilonidal, sea esta complicada o no. Se presentan 3 casos clínicos resueltos mediante esta técnica.

Materiales y método: Se presentan tres casos clínicos, operados entre 01/03/2019 y 01/08/2019 en Hospital Interzonal General de Agudos San Roque de Gonnet (División Coloproctología), de pacientes que presentaban enfermedad pilonidal crónica con episodios de reagudización y sometidos a distintos tratamientos médicos y quirúrgicos previamente. Todos ellos de la línea media y de tamaño grande. Dos casos de sexo masculino y uno de sexo femenino. Se presenta la evolución de cada uno de ellos con seguimiento a nueve meses.

Conclusiones: El colgajo de Dufourmentel representa una alternativa viable frente a quistes sacrococcígeos grandes, por dentro o por fuera de la línea media. Por su fácil confección y sus buenos resultados debería estar dentro de las opciones del cirujano general para el tratamiento de la enfermedad pilonidal.
\end{abstract}

Palabras claves: Enfermedad pilonidal; Dufourmentel; Técnica

\begin{abstract}
Introduction: The sacrococcygeal pilonidal disease (SPD) is a frequent condition described for the first time in May, 1833. Its course is benign (only $0.1 \%$ becomes malignant giving rise to a squamous cell carcinoma). Recurrence is variable according to different series and the type of surgical procedures and the associated morbidity is high. A number of surgical techniques are described for its resolution.

Objective: To describe the use and results of the Dufourmentel flap for the treatment of complicated or uncomplicated SPD. Material and methods: Three consecutive patients (2 males) with recurrent exacerbated episodes of chronic SPD, who underwent resection and primary closure with a Dufourmentel flap during January 2019 at the Coloproctology Division, Hospital Interzonal General de Agudos San Roque de Gonnet are presented. All of them had previously undergone different medical and surgical treatments.

Results: All the lesions were large and located in the midline. The flap preparation was straightforward. The postoperative course was uneventful and all patients healed without dehiscence or suppuration of the flap within 12 postoperative days. There were no recurrences with a maximum follow-up of 9 months.

Conclusion: The Dufourmentel flap was a valuable alternative for the treatment of large recurrent sacrococcygeal cysts. Due to its easy preparation and good results, it should be within the options of the general surgeon and the coloproctologist for the treatment of pilonidal disease.
\end{abstract}

Keywords: Pilonidal disease; Dufourmentel flap; Technique

\section{INTRODUCCIÓN}

La enfermedad pilonidal sacrococcígea (EPS) es una afección aguda o crónica, que suele afectar la región sacrococcígea de los adultos jóvenes. Se trata de una afección frecuente (26/100000) con una relación hombre/mujer de 2,2:1 1 y que aparece descripta por primera vez por mayo en $1833 .^{2}$ Es de curso benigno y solo $0,1 \%$ se maligniza a carcinoma escamoso. ${ }^{3}$

\section{Sergio Schlain}

sergiofschlain@gmail.com

Recibido: marzo de 2020. Aceptado: agosto de 2020

Los autores declaran no tener ningún conflicto de interés.
Muchas veces menospreciada, toma valor cuando analizamos los datos que presenta en cuanto a morbilidad, tanto del curso de la enfermedad como de su postoperatorio. Presenta una amplia y variada gama de técnicas para su tratamiento, todas ellas, con tasas sumamente variables de recurrencias que van del 1 al 28,5 \%. ${ }^{4}$

Se describen como posibilidades terapéuticas operaciones no resectivas, desde el destechamiento, la operación de Buie o la marsupialización, hasta la resección total de la misma y la reparación del defecto con un colgajo.

El paradigma pareciera ser la cirugía resectiva, ya que ofrecería la cura completa de la enfermedad. El inconveniente que presenta es el cierre del defecto, muchas veces 
a tensión, con el fracaso del mismo y el cierre por segunda intención. Como alternativa de esto han surgido las opciones no resectivas con el objetivo de mejorar los postoperatorios con una mejora en los cierres.

La mención de tantos y tan variados procedimientos nos da la pauta de que ninguno de ellos cumple con los requisitos para transformarse en el gold standard para el tratamiento. Todo ese abanico de procedimientos se ha ido utilizando y modificando en pos de objetivos comunes:

1. acortar estadía hospitalaria,

2. acortar el tiempo de cicatrización con disminución de las complicaciones,

3. acortar el tiempo de retorno a las actividades habituales y

4. lograr el menor índice de recidivas.

Cuando se analizan los datos de recurrencia se ven múltiples factores o variables que en ellos influyen. Estas variables tienen que ver con el tipo de cirugía realizada, el lugar donde se efectuaron los procedimientos y la experiencia del equipo quirúrgico tratante,,${ }^{5}$ entre otros.

\section{OBJETIVO}

El objetivo de la presente publicación es presentar una serie de casos donde se muestra la utilización de un procedimiento resectivo con cierre primario del defecto mediante colgajo. El colgajo que utilizamos para esta serie es el denominado de Dufourmentel y lo consideramos una alternativa válida para el tratamiento de la EPS, sea esta complicada o no.

\section{MATERIALES Y MÉTODO}

Para este trabajo presentamos 3 casos que fueron operados entre el 01/03/2019 y 01/08/2019 por la División de Coloproctología del Hospital Interzonal General de Agudos San Roque, de Gonnet (Hospital dependiente del Ministerio de Salud Pcia. Bs. As.).

Se presentan dos casos de pacientes masculinos y uno de una paciente femenina.

Los pacientes fueron seguidos por nueve meses con controles clínicos sin presentar complicaciones (evaluadas mediante la escala de Dindo-Clavien). ${ }^{6}$ Se puso especial atención en los defectos de cierre, hipoestesias (permanentes o transitorias) y recidivas. El control clínico se realizó en consultorio externo y cada 7 días durante el primer mes. Luego una vez por mes hasta completar los nueve meses de postoperatorio.

Se indicó el uso de rasurado postoperatorio, el uso cremas depilatorias a partir del día 15 y la depilación definitiva tras seis meses.

\section{Descripción técnica}

La técnica comienza con la demarcación del rombo de resección y de los futuros flaps. El rombo de resección se delimitará con los vértices A-B-C-D, como lo muestra el gráfico 1 .

Abarca la lesión quística (seno principal) y las posibles fístulas, pasando por sectores de piel sin compromiso inflamatorio.

Los flaps se demarcan de la siguiente manera (gráfico 2):

1. Se prolonga la línea imaginaria $(\mathrm{A}-\mathrm{C})$ que divide el rombo de resección en 2 triángulos equiláteros con líneas punteadas.

2. Se prolonga la línea o lado $\mathrm{D}-\mathrm{C}$ del rombo, también con línea punteada.

3. Se traza la bisectriz del ángulo formado por estas 2 prolongaciones.

4. Estas líneas prolongadas tendrán la longitud de las líneas originales del rombo.

Finalmente se demarca el lado externo del colgajo que se diseña, trazando una perpendicular a la prolongación A-C (perpendicular a la línea punteada) y que se llamará línea E-F, como lo muestra el gráfico 3.

Sigue a esto el tiempo de resección romboidal del quiste junto con todas las lesiones visibles. La resección debe profundizarse hasta la aponeurosis presacra.

Tras la resección completa, se incide sobre las líneas C-E y E-F confeccionando los flaps del colgajo de rotación, también hasta la aponeurosis.

La transposición del colgajo se realiza uniendo el punto $1 \mathrm{C}$ con $\mathrm{A}$ y el punto $2 \mathrm{E}$ con $\mathrm{B}$ pivoteando el flap de rotación sobre el punto D como lo muestra el gráfico 4.

La fijación de los flaps se realiza con puntos separados de una sutura reabsorbible (poliglactina 910, 1) a la aponeurosis sacra y con tejido celular subyacente. La hemostasia se realiza exhaustivamente con electrocauterio monopolar.

Para la sutura de piel se puede utilizar nylon 3-0.

En ningún caso de la serie se colocó drenaje postoperatorio. Se administraron cefalosporinas en la inducción anestésica y solo se mantuvo la indicación en el caso supurado, por 5 días vía oral. En todos los casos se realizó la operación bajo anestesia regional y se indicó la ambulación postoperatoria inmediata.

\section{RESULTADOS}

\section{Presentación de casos}

\section{Caso 1}

Paciente femenina, de 43 años, que consulta por EPS de 4 años de evolución. Presenta como antecedentes de enfermedad actual múltiples tratamientos con antibióticoterapia y tres drenajes quirúrgicos en los últimos 3 años. Se realizó resección y cierre primario mediante colgajo de Dufourmentel. Se le dio el alta a las $8 \mathrm{~h}$ del procedimien- 


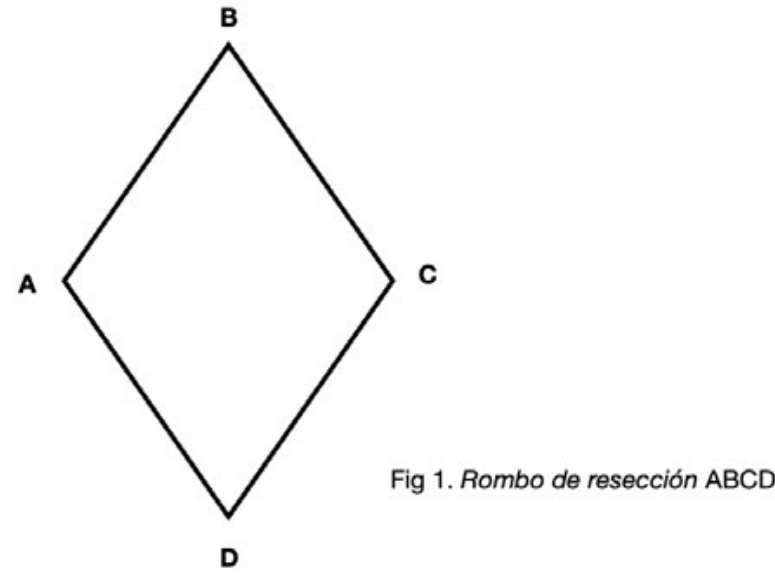

Gráfico 1: Rombo de resección A-B-C-D.

A



Gráfico 2: Demarcación inicial de los flaps de rotación.

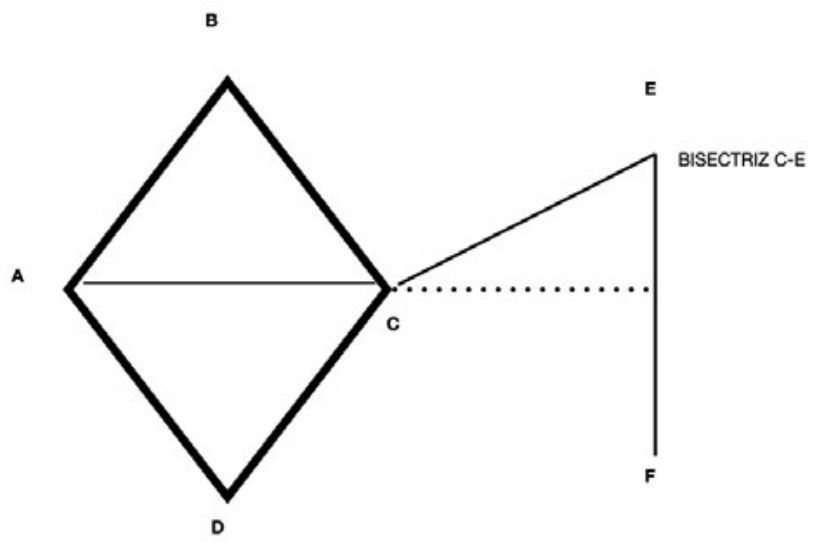

Gráfico 3: Configuración final de la demarcación del colgajo.

to. Se retiraron puntos al día 12 del postoperatorio.

La paciente retomó su actividad laboral al quinto día del postoperatorio.

En el control de 30 días presenta cierre completo (figuras 1 a 3 , caso 1 ).

\section{Caso 2}

Paciente masculino, de 25 años de edad, que consulta por EPS en fase supurativa de 20 días de evolución, que res-


Gráfico 4: Transposición del colgajo.

ponde parcialmente al tratamiento médico antibiótico con ciprofloxacina. Tiene antecedentes de episodios reiterados e intermitentes desde hace aproximadamente 8 años. Se realizó resección y cierre primario con colgajo de Dufourmentel.

Se le dio el alta al día 1 del postoperatorio.

Retomó sus actividades laborales al séptimo día del postoperatorio.

Se retiraron puntos al décimo día del postoperatorio.

En el control de 30 días no presentó alteraciones (figuras 4 a 8 , caso 2).

\section{Caso 3}

Paciente masculino, de 25 años de edad, que consulta por EPS en etapa supurativa de $10 \times 7 \mathrm{~cm}$, con 5-6 años de evolución. Se indicó Ciprofloxacina $500 \mathrm{mg}$ cada $12 \mathrm{~h} \mathrm{du}-$ rante los 10 días previos a la cirugía, por encontrarse en estado supurativo.

Se realizó resección romboidal y cierre primario mediante colgajo. Tuvo una buena evolución postoperatoria.

Se le dio el alta al segundo día del postoperatorio.

Se retiraron puntos a los 12 días del postoperatorio.

Se reincorporó a su actividad laboral a los 15 días del postoperatorio (figura 9 a 12, caso 3).

\section{DISCUSIÓN}

La EPS es una entidad frecuente que, por razones desconocidas, ha aumentado su incidencia en forma continua durante los últimos 50 años, particularmente en hombres jóvenes europeos y norteamericanos. En una cohorte militar alemana, por ejemplo, el número de pacientes afectados aumentó de 29/10000 en el año 2000 a 48/100000 en el año 2012, y el número total de cirugías, en Alemania, en pacientes con EPS superó el número de intervenciones relacionadas a hernias inguinales en el rango etario de 20 a 40 años. $^{7}$

La descripción de técnicas, procedimientos y variaciones de los mismos es innumerable, teniendo en un extremo la apertura del seno solamente hasta, en el otro extremo, la utilización del láser. Todos estos procedimientos se reali- 




Figura 1: Demarcación de rombo de resección A-B-C-D. Se demarca también la prolongación en punteado de D-C, la prolongación de A-C, y la bisectriz de este ángulo 1-2. E-F es perpendicular a A-C.

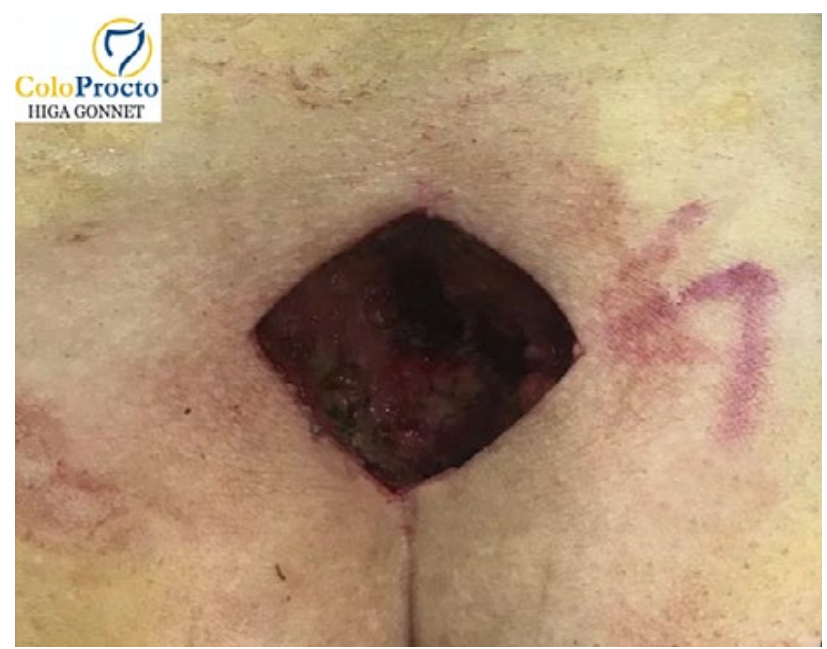

Figura 2: Resección de la zona enferma.

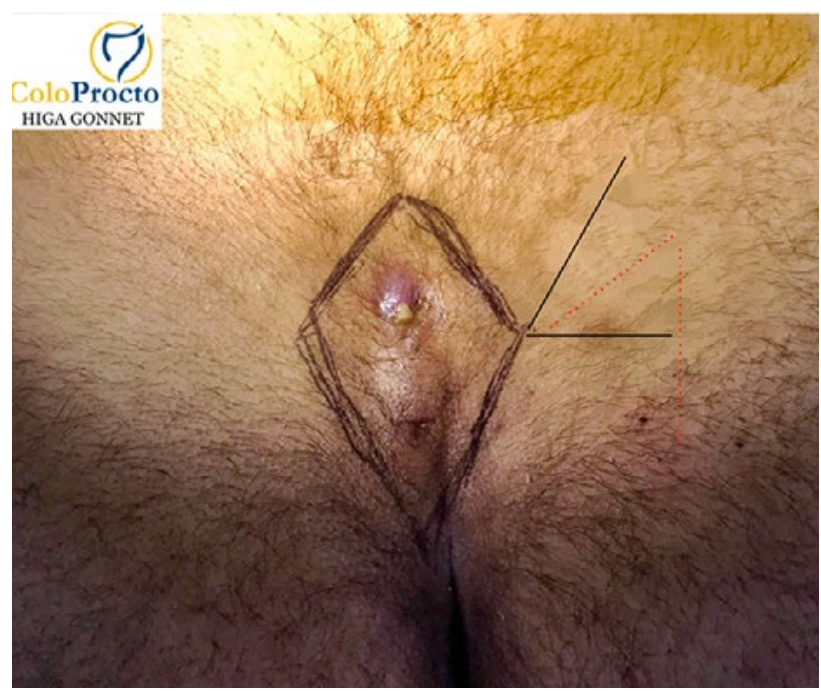

Figura 3: Cierre primario.

zan en busca de objetivos comunes a la terapéutica, como lograr un procedimiento completo (extracción de todos los senos y fístulas) rápido, sencillo, de bajo costo, que nos

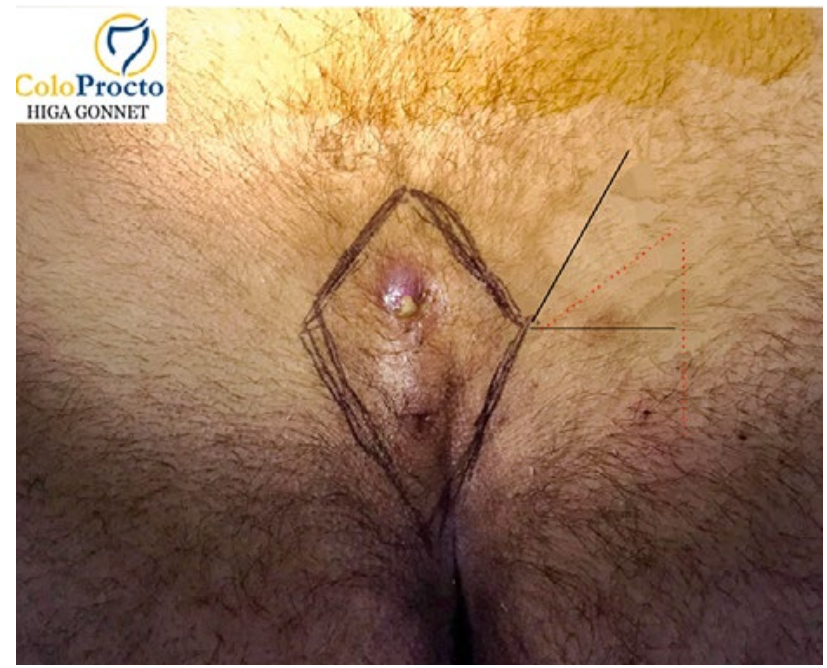

Figura 4: Lesión superlativa. Se demarca el rombo de resección y se muestran las demarcaciones que serán líneas de sección en la configuración de los colgajos.

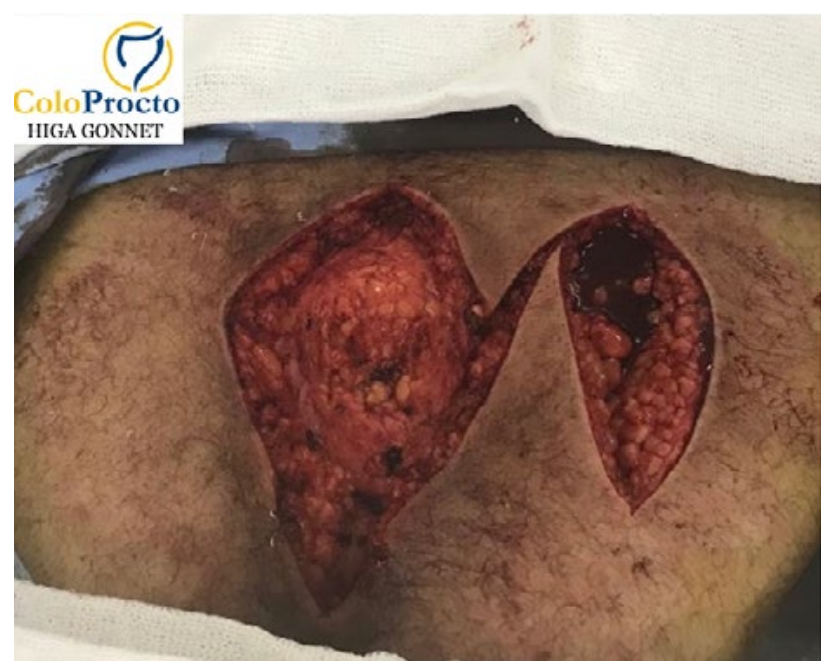

Figura 5: Resección completa de la lesión supurativa, complejo quiste-fístulas y confección de las incisiones, siguiendo las líneas preparadas.



Figura 6: Presentación de la transposición de los colgajos.

permita una corta estadía hospitalaria con rápido retorno a la actividad habitual, bajo índice de complicaciones y una tasa de recurrencias lo más baja posible. En esta búsqueda del "gold standard" es que la literatura genera datos 


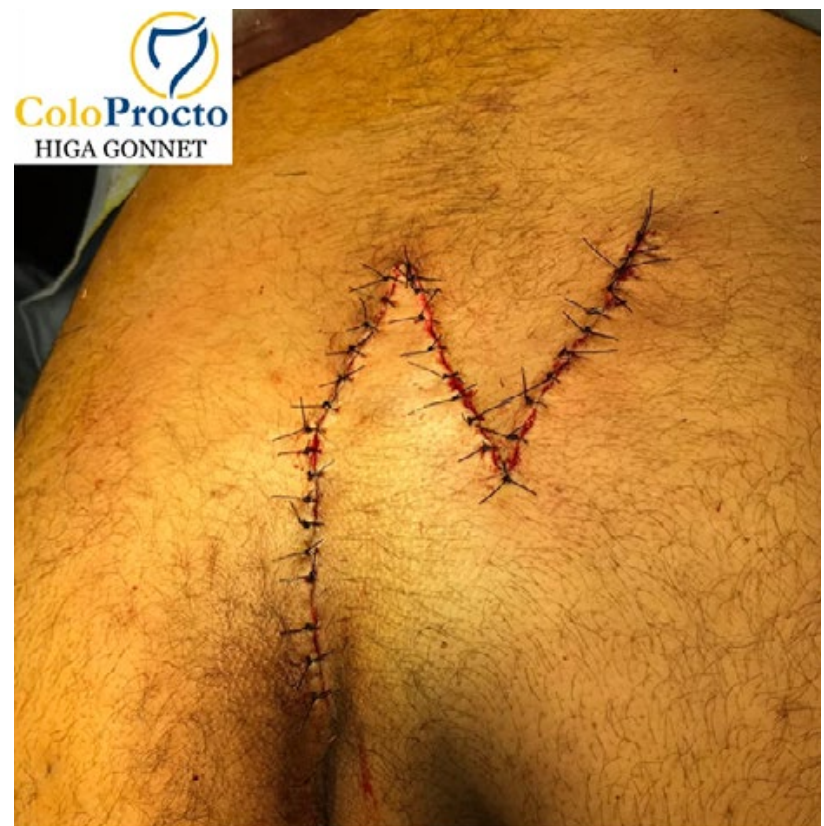

Figura 7: Cierre de piel al final de la operación.

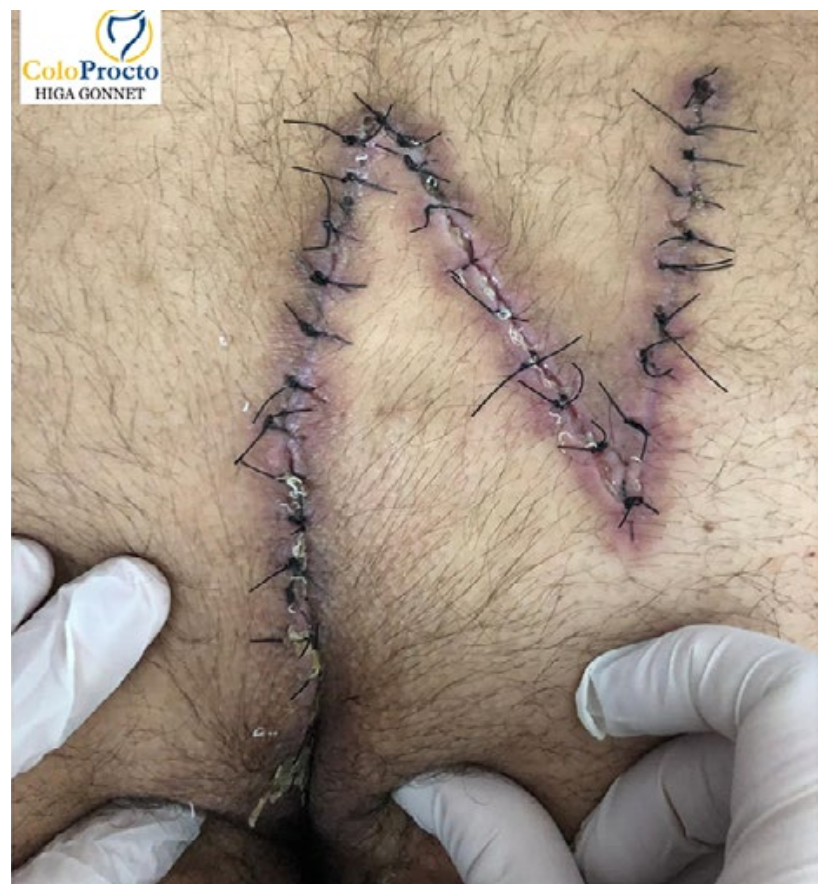

Figura 8: Control clínico al día 11 del postoperatorio.

encontrados. Quizá esto se deba a la heterogeneidad que se presenta en los seguimientos. En este punto es donde debemos ser críticos sobre nuestro trabajo. Presentamos un seguimiento corto de los pacientes como para tomar decisiones o inferir resultados sobre recidivas. En relación a las recidivas, y en uno de los pocos metaanálisis publicados, Stauffer (2018) presenta datos a 12 meses y las tasas más bajas de recurrencias las aportan los flaps de Limberg y Dufourmentel (0,3 \%). ${ }^{8}$ Estos números se mantienen como los más bajos también a los 24 meses (1,6 \%).

El problema que se le atribuye al clásico colgajo de Lim-

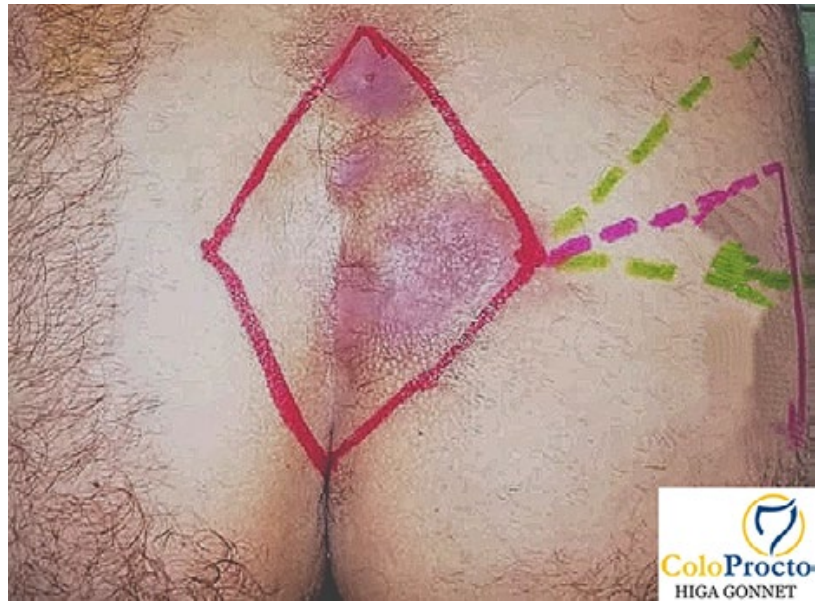

Figura 9: Lesión sobreinfectada grande (10 x $7 \mathrm{~cm}$ aproximadamente), y demarcación de los colgajos.



Figura 10: Resección romboidal completa de la formación (quiste-fístulas).



Figura 11: Confección de los colgajos.

berg es la cicatrización relativamente pobre de la herida, particularmente en el polo inferior del colgajo, cerca del canal anal, donde se puede observar una grave maceración y dehiscencia de la herida por la rotación del colgajo y la tensión de la sutura. ${ }^{9}$ Por esto se han buscado alternativas al mismo, manteniendo el mismo concepto, y es así que surge la modificación de Dufourmentel. Tiene la ventaja de una base más amplia con un área de rotación más pequeña ${ }^{10}$ tal como lo demostramos en la descripción técnica y en el gráfico 5 . 


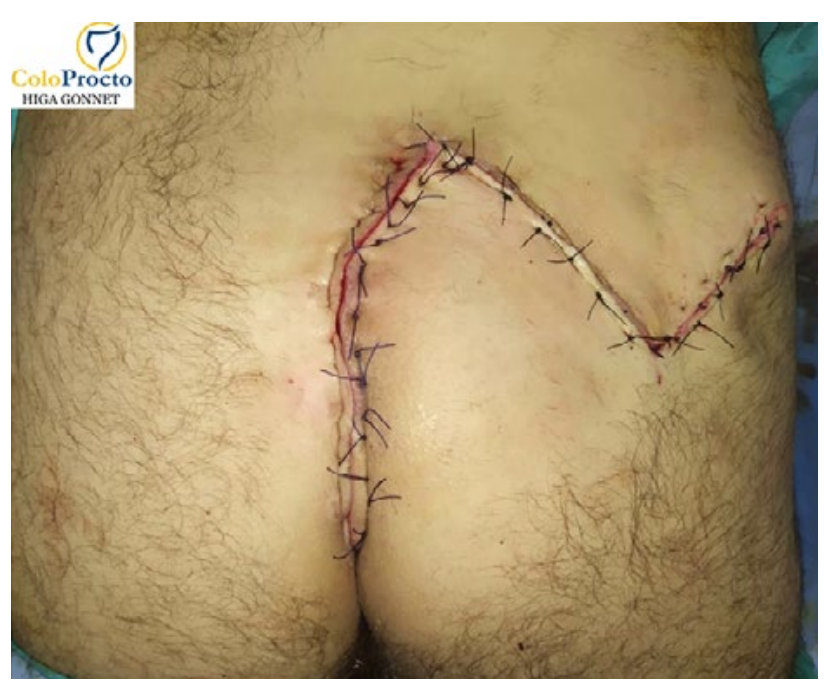

Figura 12: Fin del procedimiento con la sutura de piel.
COLGANO DE LIMBERG



Base del Colgajo



Base del Colgajo
Gráfico 5: Comparación entre la base del colgajo de Limberg y la del Dufourmentel Un estudio de Lieto, quien presenta 310 pacientes con EPS tratada mediante colgajo de Dufourmentel, muestra una baja tasa de recurrencia $(97,6 \%$ de los pacientes sin recurrencias) a 16 años. ${ }^{12}$

Ishii (2017) presentó una serie de casos con seguimiento a 4 años donde ninguno de sus pacientes desarrolló necrosis del colgajo y solo uno mostró una dehiscencia. Ninguno presentó hipoestesias ni recurrencias. ${ }^{11}$

Un estudio de Lieto, quien presenta 310 pacientes con EPS tratada mediante colgajo de Dufourmentel, muestra una baja tasa de recurrencia $(97,6 \%$ de los pacientes sin recurrencias) a 16 años. ${ }^{12}$

Los datos de recurrencias en general son muy varia- dos y los estudios en su mayoría (por el tipo de diseño) no muestran los cuidados postoperatorios que recibieron los pacientes (protocolo de Harris). Tampoco muestran si recibieron rasurados o depilación de la zona afectada y, si lo hacen, son muy variables en el tipo de depilación. ${ }^{13,14} \mathrm{La}$ depilación postoperatoria con láser parece mostrar mejoras en los resultados de recurrencia y se están desarrollando protocolos para su implementación. ${ }^{15}$

La principal crítica que nos hacemos es el corto seguimiento de nuestra serie para la evaluación de recurrencias, pero tras 9 meses no ocurrieron. Lo que sí mostramos, y que creemos es para destacar, es una corta estancia hospitalaria de los pacientes, con una rápida vuelta a las actividades habituales, sin importar si las mismas conllevaban o no esfuerzo físico y sin complicaciones locales asociadas al procedimiento como hipoestesias permanentes ni necrosis de los colgajos realizados. La única desventaja de este procedimiento es el resultado cosmético, que puede no ser atractivo.

Teniendo en cuenta la ubicación de la enfermedad, la curación de heridas por primera intención y un retorno temprano a la actividad completa, los factores cosméticos no son de relevancia y las ventajas superan un resultado cosmético desfavorable. ${ }^{16}$

\section{CONCLUSIONES}

El colgajo de Dufourmentel representa una alternativa viable frente a la EPS con quistes grandes, por dentro o fuera de la línea media, estén o no complicados.

Por su fácil confección y sus buenos resultados debiera estar dentro de las opciones del cirujano general y del coloproctólogo para el tratamiento de la EPS, ya que ofrece la posibilidad de realizar la resección completa del complejo quiste-fístula con cierre primario sin tensión. Al lograr esto ofrece la posibilidad de una corta estancia hospitalaria y un retorno más rápido a la actividad habitual, sin complicaciones.

\section{REFERENCIAS}

1. McCallum I, King PM, Bruce J. Healing by primary versus secondary intention after surgical treatment for pilonidal sinus. Cochrane Database Syst Rev 2007;4:CD006213.

2. Mayo $\mathrm{OH}$. Observations on injuries and diseases of the rectum. London: Burgess and Hill; 1833. p. 45-6.

3. Parpoudi SN, Kyziridis DS, Patridas DCh, Makrantonakis AN, Iosifidis P, Mantzoros IG, et al. Is histological examination necessary when excising a pilonidal cyst?. Am J Case Rep 2015;16:164-68.

4. Larsson JC, Dieguez Aliaga F, Granero L, Cornet M. Tratamiento de la enfermedad pilonidal crónica. Comparación de 3 técnicas quirúrgicas. Rev Argent Coloproct 2014;25:64-70.

5. Doll D, Orlik A, Maier K, Kauf P, Schmid M, Diekmann M, et al. Impact of geography and surgical approach on recurrence in global pilonidal sinus disease. Sci Rep 2019;9:15111. doi: 10.1038/s41598- 019-51159-z.

6. Dindo D, Demartines N, Clavien PA. Classification of surgical complications: a new proposal with evaluation in a cohort of 6336 patients and results of a survey. Ann Surg 2004;240:205-13.

7. Luedi MM, Kauf P, Evers T, Sievert H, Doll D. Impact of spinal versus general anesthesia on postoperative pain and long term recurrence after surgery for pilonidal disease. J Clin Anesth 2016;33:236-42.

8. Stauffer VK, Luedi MM, Kauf P, Schmid M, Diekmann M, Wieferich $\mathrm{K}$, et al. Common surgical procedures in pilonidal sinus disease: A meta-analysis, merged data analysis, and comprehensive study on recurrence. Sci Rep 2018;8:3058.

9. Kaya B, Eris C, Atalay S, Bat O, Bulut NE, Mantoglu B, et al. Modified Limberg transposition flap in the treatment of pilonidal sinus disease. Tech Coloproctol 2012;16:55-9. 
10. Sebastian M, Sroczyński M, Rudnicki J. The Dufourmentel modification of the Limberg flap: Does it fit all? Adv Clin Exp Med 2017;26:63-7.

11. Ishii N, Shimizu Y, Oji T, \& Kishi K, Modified Dufourmentel flap with superior pedicle: a useful technique for sacrococcygeal pilonidal sinus. J Plast Surg Hand Surg 2017;51:453-57.

12. Lieto E, Castellano P, Pinto M, Zamboli A, Pignatelli C, Galizia G. Dufourmentel rhomboid flap in the radical treatment of primary and recurrent sacrococcygeal pilonidal disease. Dis Colon Rectum 2010;53:1061-68.

13. Petersen S, Wietelmann K, Evers T, Hüser N, Matevossian E, Doll
D. Long-term effects of postoperative razor epilation in pilonidal sinus disease. Dis Colon Rectum 2009;52:131-34.

14. Harris C, Sibbald RG, Mufti A, Somayaji R. Pilonidal sinus disease: 10 steps to optimize care. Adv Skin Wound Care 2016;29:469凶78.

15. Minneci PC, Halleran DR, Lawrence AE, Fischer BA, Cooper JN, Deans KJ. Laser hair depilation for the prevention of disease recurrence in adolescents and young adults with pilonidal disease: study protocol for a randomized controlled trial. Trials 2018;19:599.

16. El-Khadrawy O, Hashish M, Ismail K, Shalaby H. Outcome of the rhomboid flap for recurrent pilonidal disease. World J Surg 2009;33:1064-68.

\section{COMENTARIO}

Refrescante presentación de casos clínicos de enfermedad pilonidal sacrococcígea con quistes de gran tamaño, resueltos con resección y colgajo de Dufourmentel. Los autores muestran, detalladamente y con buenas imágenes, esta técnica quirúrgica de realización poco frecuente pero que ha demostrado tener buenos resultados.

Si bien el número de casos es pequeño y el tiempo de seguimiento clínico es relativamente corto, este artículo aporta una descripción detallada de esta técnica quirúrgica, acompañada de una breve revisión bibliográfica que colabora para tenerla presente como alternativa terapéutica ante estos casos.

Como bien describen, no existe técnica Gold Standard, sino que el tratamiento quirúrgico de esta patología debería personalizarse, teniendo en cuenta las particularidades tanto de la lesión como así también del paciente. Felicitaciones a los autores.

Sanatorio Sagrado Corazón y Sanatorio Finochietto, Ciudad Autónoma de Buenos Aires, Argentina. 